\title{
Kaon Factories
}

\author{
F. Bradamante \\ Dipartimento di Fisica, Universita di Trieste \\ and Sezione di Trieste dell'INFN, Italy
}

\begin{abstract}
Beams of secondary particles such as kaons allow a rich and diversified experimental programme in particle and nuclear physics, complementary to the one pursued at very high energy accelerators.
\end{abstract}

The production of intense beams of kaons to systematically study its rare decay modes was considered in the late 1970's of such importance as to justify the construction of dedicated accelerators. Interest in the kaon system had persisted right from the earliest beginnings of particle physics, as it was, and indeed remains, a source of fundamental insights into new physics (e.g., the $\tau-\theta$ puzzle, the $\Delta /=1 / 2$ rule, $c p$-violation, and the absence of strangeness-changing neutral currents). This explains why kaons were singled out in naming "factories" comprising proton accelerators in the $10-60 \mathrm{GeV}$ energy range with a 10-100 $\mu \mathrm{A}$ proton beam current, specifically designed to produce beams of secondary particles (neutrinos, pions, muons, kaons, antinucleons, and hyperons) but at higher intensities or with higher purities than were currently available.

I shall review the physics case for kaon factories by discussing objectives common to the huge variety of important experiments intrinsic to these facilities: the present status of international projects and European initiatives are outlined separately on pages 8 and 9, respectively.

\section{Physics at Kaon Factories}

The description of the sub-atomic world in terms of leptons and quarks, interacting via gluons and electroweak bosons in the framework of the Standard Model of particle physics, represents one of the superb intellectual achievements of the last few decades. However, in spite of its many successes, the model is not regarded as a complete theory: the feeling is widespread that there is new physics to be discovered, and that to this end both new theory and new experimental data are needed.

Three different arenas are being explored in a monumental world-wide effort: - the energy frontier, now ranging between 0.2 and $20 \mathrm{TeV}$, involving fundamen-

Franco Bradamante was appointed Professor of Particle Physics at the University of Trieste, Via A. Valerio, 2, I-34127 Trieste in 1980 after holding temporary posts. He studied mainly at Trieste, receiving his Ph.D. in 1963 before taking up a Fullbright Fellowship at Cornell in the USA. His experimental work, mostly at CERN, over the last 10 years has largely involved low-energy baryon interactions. tal issues such as the Higgs boson, $\mathrm{W}$ and $Z$ particle physics and supersymmetric quarks and leptons;

- passive physics addressing phenomena such as monopoles, proton decay, solar neutrinos, and neutrino oscillations, at large underground detectors;

- the precision frontier looking towards rare and forbidden processes, $\mathrm{cp}$-violation, hadron dynamics and spectroscopy, and neutrino physics, at existing accelerator facilities such as CERN's Large Electron-Positron (LEP) collider.

It is impossible to predict today in which of the three arenas the breakthrough to a new physics will occur: they are complementary, and are being investigated in parallel. Kaon factories are intermediate energy accelerator facilities optimized for problems arising in the third arena.

The physics case for kaon factories, spelt out almost ten years ago, has been discussed at an incredible number of meetings and can be found in the various project proposals (see page 8 and [1]). Specific topics are: rare k-decays and $\mathrm{cp}$ violation; neutrino physics; hadron spectroscopy; hyperon physics; polarization and symmetries; polarization and hadron dynamics; hypernuclear physics and exotic atoms; kaon scattering and spectroscopy; antiproton physics; and low energy muon science.

Each of these topics underlines a major area of investigation where activity has gone on over many years, and where further progress calls for precision experiments, i.e., beams of higher intensity to access rare phenomena and to look for weak signals. Clear-cut advances are virtually guaranteed by kaon factories as they produce secondary beams a factor of 100 times more intense than the best beams available today (at Brookhaven National Laboratory's AGS ring in the USA).

The factor of 100 is not a magic number, but represents a satisfactory compromise between the physics needs (covering as many areas as possible), present-day technology (handling high intensity beams poses some problems) and obvious budgetary constraints. The factor of 100 is a reference figure, and as such has been adopted in all proposals for new facilities.

\section{Two Common Denominators}

If common denominators were to be identified in the physics objectives of kaon factories, I would like to isolate just two: i) The dynamics of quarks and gluons is still not described by a complete and selfconsistent theory. The theory of quantum chromodynamics (QCD) is surely an important step, but as so many problems (notably confinement) are either unresolved or cannot be calculated, the strong interactions are still an open problem. Vigorous experimental programmes on hadron spectroscopy and reaction mechanisms, with particular attention given to the effects of spin, have been proposed, as well as selected experiments using nuclear targets to probe confinement in the nuclear medium.

ii) The existence of several families of quarks and leptons is a puzzle. The dynamical origin of the masses of the fermions, the only parameter differentiating the various families, is completely unknown. Searching for the presence of high masses in rare or forbidden kaon decays is probably a very good way to obtain signals heralding a new physics. Also, the discovery of neutrino oscillations would provide an important step towards grand unification schemes.

An unique "magic experiment" cannot be proposed today to solve these two problems, but the variety of experiments intrinsic to a kaon factory is the best guarantee of important findings and possibly breakthroughs in physics.

\section{Colour Physics}

The study of hadronic interactions in the confinement regime, i.e., colour dynamics, has become a research area of common interest to both nuclear and particle physicists, who speak in much the same language today as opposed to, say, 10 years ago. In this sense, a kaon factory will provide a bridge between low energy particle physics and nuclear physics with high energy hadronic probes, and will strengthen common research efforts, to the benefit of both.

Probing the confinement mechanism and its associated phenomena can only be carried using a kaon factory's hadron beams. Empirically, confinement of the 
basic degrees of freedom is a unique property of strong interactions, although it is believed theoretically that this property is shared by gauge theories beyond QCD. However, experimental investigations of the confinement mechanism seem to be feasible only in the context of strong interaction physics.

There is numerical evidence from lattice gauge calculations that QCD does indeed exhibit the property of confinement, but it is not clear theoretically how confinement arises, nor are the phenomenological implications of confinement fully understood. For instance, it is not known whether the confining forces saturate at the most elementary level, giving rise only to the ordinary hadrons as both quark-antiquark and three-quark systems, or whether these forces allow for exotic multiquark states or resonances. Similarly, there is very little understanding of the rôle of the gluons: approaches such as the non-relativistic quark model which completely ignore the confined gluon degrees of freedom are remarkably successful in the description of hadronic spectra. Still less complete is our understanding of hadron-hadron interactions in terms of the basic degrees of freedom.

It should be stressed at this point that there is a tendency to put aside this whole domain of physics as a kind of "chemistry" of strong interactions, too complex and too difficult to be tractable, and to concentrate on "asymptotic" physics at the highest energies. In this line of thought, non-perturbative quantum chromodynamics is looked at with suspicion, and the hard problems of $Q C D$ are believed to be best approached through largescale computer calculations, i.e., by means of Monte-Carlo simulations of local gauge theories on lattices. Personally, I do not consider this solution to the problem of strong interactions to be satisfactory, and I believe our goal should still be to calculate in a simple way quantities such as the mass of the pion, as was done by Rydberg at the beginning of this century.
To this end, hints from Nature are essential, and to have available a tool capable of producing a significant quantity of hadronic data seems a necessary step towards understanding hadronic matter.

\section{Rare kaon decays}

There is overall agreement within the scientific community of the very special rôle played by the kaon and of the importance of studying its rare decay modes. For instance, a low energy proton synchrotron (ASTOR) was proposed several years ago for SIN, now the Paul Scherrer Institute in Villigen, Switzerland, specifically to produce low energy kaon beams (Ed. : an alternative proposal for a beauty factor was eventually taken up for further study, but the project was not funded).

The study of rare kaon decays shows some parallels to the study of rare muon decays at meson factories, and it is worthwhile to recall that the today's best value to the limit for the existence of righthanded weak currents is given by preci-

\section{TRIUMF Leads the Way}

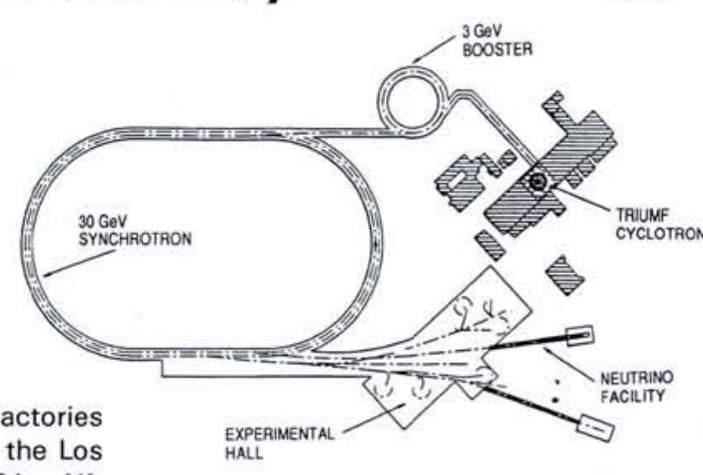

Five separate proposals for kaon factories Alamos National Laboratory, USA, [1] TRIUMF, Vancouver, Canada [2] , the European Hadron Facility (EHF) Study Group [3], the Soviet Union [4] and Japan [5]. At present, the Canadian KAON project is closest to final approval. A two-year pre-construction study was funded jointly by the Province of British Columbia and the Canadian federal government in early 1988 . The federal government matched, last October, the provincial government in agreeing to $\mathrm{fi}$ nance one-third of the construction cost: international support (mainly from the USA and Japan) will hopefully provide the rest.

$\mathrm{KAON}$ (see figure) aims for a $100 \mu \mathrm{A}$ proton beam accelerated to $30 \mathrm{GeV}$, using TRIUMF's existing cyclotron as injector. A $3 \mathrm{GeV}$ booster synchrotron as an intermediate accelerating stage is necessary, as well as a dedicated stretcher ring at $30 \mathrm{GeV}$ to allow for direct current beam extraction.

In spite of the many years hard work and the enthusiastic effort of hundreds of physicists, proposals for the Advanced Hadron Facility at Los Alamos National Laboratory, USA, and for the European Hadron Facility (see page 9) have not been approved.

The Japanese Hadron Project is a proposal for the construction, in the long term, of an accelerator complex at KEK, Tsukuba.
The first stage comprises a $1 \mathrm{GeV} / 200 \mu \mathrm{A}$ proton linac and a $1 \mathrm{GeV}$ compressor ring. Kaon physics will be pursued by injecting part of the proton beam directly into the existing KEK proton synchrotron and accelerating it to $12 \mathrm{GeV}$. The proposal also calls for the construction of a heavy ion linac to accelerate well above the Coulomb barrier the exotic nuclei produced by irradiating a thick target with the proton beam from the linac, thus providing radioactive beams of highly unstable nuclei (i.e., far from the $\beta$ stability line) to study nuclear matter under extreme conditions. A second stage involving the construction of a new $30 \mathrm{GeV} / 100$ $\mu \mathrm{A}$ proton synchrotron is also envisaged. $\mathrm{A}$ characteristic feature of the project is that it addresses both nuclear and particle physics and materials science, by providing pulsed muon and neutron beams in very short spills (20 ns). The project is promoted by the Institute for Nuclear Studies; approval for the first stage was expected in 1991.

The Moscow Kaon Factory is an approved long-term project of the Institute for $\mathrm{Nu}$ clear Research. It consists of an accelerator complex to accelerate to $30 \mathrm{GeV}$ the proton beam delivered by a $600 \mathrm{MeV}$ linac being commissioned for a meson factory.
To these dedicated projects one should add the upgrading programmes being undertaken in the USA at the Brookhaven $\mathrm{Na}$ tional Laboratory (BNL) [6] and at the Fermi National Accelerator Laboratory [7]. For the first, commissioning of a booster ring started last spring and the ring should be operational this summer, providing a 4-6 times more intense proton beam than BNL's AGS ( $\approx 20$ times less than KAON). A stretcher ring was proposed to the US Department of Energy (DoE) but the financial situation is such that it is unlikely to be funded. For the second, a proposal has been submitted to the DoE for the construction of a new Main Injector to the Tevatron accelerator which, if accepted, would provide the most powerful source of high energy kaon beams in the world. The DoE decided last summer that it would not fund the upgrade in fiscal year 1992, but would continue to consider the project.

[1] A Proposal to Extend the Intensity Frontier of Nuclear and Particle Physics to 45 GeV (LAMPF II), LA-UR-84-3282 (Los Alamos National Laboratory, USA) 1985.

[2] KAON Factory Proposal (TRIUMF, Vancouver, Canada)1985; Kaon Factory Study (TRIUMF) 1990.

[3] Proposal for a European Hadron Facility, Ed. J. Crawford, EHF-87-18 (1987).

[4] Chursin A.G. et al., The Accelerator Complex of a Kaon Factory of the INR of USSR, Proc. XI All Union Conf. on Accelerators for Charged Particles (1988).

[5] Kihara M., Outline of the Japanese Hadron Facility, KEK Preprint 86-106 (1987). [6] Foelsche H.W.J., BNL Upgrade Plans, in Proc. of the Int. Workshop on Hadron Facility Technology, Ed. H.A. Thiessen, LA11130C (Los Alamos National Lab, USA) 1987. [7] Fermilab Upgrade: Main Injector - Conceptual Design Report, Project No. 91-CH 400 (Fermi National Accelerator Laboratory, USA) 1989. 
sion experiments for the $\mu^{+} \rightarrow \mathrm{e}^{+} v \bar{v}$ decay at TRIUMF in Canada [2]. On the other hand, since the kaon belongs to the second generation of fundamental particles, the variety of decay channels accessible to kaons is much larger than in the case of the muons, and the physics potential is much richer. Moreover, $\mathrm{cp}$-violation manifests itself in the kaon system. Although very accurate experiments are presently being performed, the study of $\mathrm{cp}$-violation in the kaon system will surely be pursued in future hadron facilities. It will, however, be a real challenge to design a significantly more accurate experiment.

Rare and ultra-rare processes, when seen in the light of the Standard Model, can be divided into two classes:

- processes which are strictly forbidden by the model and whose existence would provide unambiguous hints for new phy sics beyond the model;

- processes which are not forbidden by the Standard Model, but which are so strongly suppressed by some mechanism that any observation at a level above the expected rate would be a signature for the model's failure.

As an example of forbidden decays, we can consider the decay $K_{L}{ }^{\circ} \rightarrow \mu^{+} \varepsilon$. This decay violates lepton number conservation so is strictly forbidden in the Standard Model. Searches of such a process are obviously very appealing because lepton violation is an automatic consequence of many of the proposed extensions of the model. If observed, its branching ratio determines a new mass scale. In analogy with the $\mathrm{K}^{+} \rightarrow \mu^{+} v$ decay mediated by the weak boson $\mathrm{W}$, one can introduce a new heavy boson $\mathrm{Y}^{\circ}$ to mediate the decay $\mathrm{K}_{L}{ }^{\circ} \rightarrow \mu \mathrm{e}$. If the coupling of this new heavy boson to the quark and to the lepton lines is similar to the weak coupling, one writes for the branching ratio $\mathrm{BR}$ : $\mathrm{BR}\left(\mathrm{K}_{\mathrm{L}}^{\circ} \rightarrow \mu \mathrm{e}\right) \sim\left(M_{\mathrm{W}} / M_{\mathrm{Y}}\right)^{4}$ where $M_{W}$ and $M_{Y}$ are the masses of the $\mathrm{W}$ and $\mathrm{Y}^{\circ}$, respectively. This decay has never been observed. An experiment at the Brookhaven National Laboratory, USA, has already provided [3] an upper limit for BR of $2.2 \times 10^{-10}$, and is aiming to push the limit down to $10^{-11}$, which would give $M_{\mathrm{Y}}>100 \mathrm{TeV}$, a mass scale inaccessible even to next-generation colliders. A kaon factory would hope to improve the limit to the branching ratio by two more orders of magnitude, mainly by increasing the beam intensity and quality.

Among the kaon decays that are not completely forbidden in the Standard Model, but are so strongly suppressed that any observation at a rate above the expected level would be a signature for its failure, particular attention has been given to $\mathrm{K}^{+} \rightarrow \pi^{+} v \bar{v}$. This decay is strongly suppressed by the GIM mechanism, first proposed by Glashow, Iliopolous and Maiani, in which the required forces cancel because all quarks appear in doublets.

\section{Significant European Interest}

The case for a European kaon factory has been promoted over several years by the European Hadron Facility (EHF) Study Group, under the leadership of F. Scheck [1]. A proposal was submitted in 1987 to the German BMFT and to the Italian INFN, the two agencies which supported the Group's activities. Two alternatives have been explored: the first involves building the EHF at a new laboratory in Italy, the second was a form of "CERN option", i.e., the possibility of realizing EHF at CERN and using existing infrastructure (specifically the ISR tunnel for housing a $30 \mathrm{GeV}$ proton synchrotron and the West Hall for experimental areas).

The cost of the full facility, built on the "green field" site, was estimated to be of the order of 800 million Swiss francs (SFR) including the basic infrastructure: a saving of about 200 M SFR could be expected for the CERN option. Neither alternative has been accepted and funding has not been granted by either INFN or the BMFT: the general feeling is that if European physicists wish to pursue kaon physics they should go to TRIUMF in Vancouver, Canada.

Given this situation it is understandable that the enthusiasm of the proponents of EHF has somewhat diminished somewhat, and that several of the scientists involved have moved to other fields.

I regard the non-approval of a project which has an excellent physics case and such a large number of supporters in Europe (estimated by the European Committee on Future Accelerators in 1986 to be more than 500 physicists) as extremely serious and a clear indication that there is a gap between the physics community and European funding institutions.

Europe has a solid tradition in hadronic physics at low energy and many groups are planning to participate in the TRIUMF experimental programme. However, it is generally felt that TRIUMF's KAON should not be the only place in the world where kaon physics is pursued, and that a hadron facility, even with a smaller beam power (e.g., a factor of 10 less), should be built in Europe.

The GIM prediction was made before the discovery of the charm quark, the doublet partner in the strange quark. The branching ratio for this decay has been estimated as long ago as 1974 [4] as $\approx 3 \times 10^{-11} \mathrm{~N}$ where $N$ is the number of generations with light neutrinos, found to be 3 at LEP and at Stanford's linear collider.

More recent calculations give $2 \times 10^{-11}$ $<\mathrm{BR}<9 \times 10^{-11}$ for $N=3$, where the uncertainty is due to the uncertainties in the mass of the top quark, in the B-meson lifetime, and in the semileptonic B-meson decay data. The importance of measuring the branching ratio of this decay is selfevident. The decay has not yet been observed, and its BR is less than $10^{-7}$. Another Brookhaven experiment is aiming for a sensitivity of $10^{-10}$, the level predicted
Ignoring opportunities at CERN, two possibilities can presently be considered for a European kaon factory. The first option is seen in the long-term future of the INFN National Laboratory at Legnaro, near Padua in Italy. The Laboratory is building and installing a superconducting RF cavities structure (ALPI) to accelerate the heavy ion beams coming from a $16 \mathrm{MV}$ Tandem Van der Graaff accelerator to energies of about $7 \mathrm{MeV} / \mathrm{amu}$ : users are arguing the case for higher energies. Two stages have been identified for a new facility, tentatively named ADRIA, the first reaching $500 \mathrm{MeV} / \mathrm{amu}$ and the second some $5 \mathrm{GeV} / \mathrm{amu}$. The second could maybe use the $9 \mathrm{GeV}$ proton booster synchrotron which has been designed for the first stage of the EHF accelerator complex, while the first would need a new pre-booster synchrotron. The goal for both stages is to accelerate all ion species, from $\mathrm{H}^{-}$to uranium, and a proposal to INFN is in preparation [2].

The second possibility is an energy and intensity upgrade of Saturne II, the $3.5 \mathrm{GeV}$ proton synchrotron at Saclay in France. Competition with other nuclear physics projects in France has so far caused this option to stay under the carpet, but the Saturne laboratory has a long tradition in intermediate energy hadron physics, and the number of people advocating such a solution is significant, as demonstrated by attendance at a workshop organized by NuPECC in 1991. However, NUPECC itself did not recommend investment in a European kaon factory in its report The Future of Nuclear Physics in Europe [EN 22 (1991) 222].

[1] Proc. Workshop on the Future of Intermediate Energy Physics in Europe, Ed. S. Galster, (KfK, Karlsruhe, Germany) 1984; Proc. Int. Conf. on a European Hadron Facility, Mainz, 1986, Ed. Th. Walcher (North Holland); Nucl. Phys. B279 (1987) Nos. 1/2. [2] Dalpiaz P. et al., Proc. 1st Workshop on Intense Hadron Facilities and Antiproton Physics, Turin, 1989, Ed. T. Bressani et al. (Editrice Compositori, Bologna).

by the Standard Model. One could reach a sensitivity of $10^{-12}$ at a kaon factory, thereby allowing accurate measurements of the decay.

\section{REFERENCES}

[1] Proc. Int. Seminar on Intermediate Energy Physics (INES-89), Moscow, 1989 (Academy of Sciences of the USSR) 1990; Proc. 18th Int. Symp. on Physics with High-Intensity Hadron Accelerators, Tokyo, 1990, Ed. S. Kubono and T. Nomura (World Scientific); Proc. Workshop on Science at the KAON Factory, Vancouver, 1990.

[2] Carr J. et al., Phys. Rev. Lett. 51 (1983) 627.

[3] Mathiazhagen C. et al., Phys. Rev. Lett. 63 (1989) 2181.

[4] Gaillard M.K. and Lee B.W., Phys. Rev. D10 (1974) 897. 